\title{
Os determinantes da inflação na República Democrática do Congo: um estudo econométrico (2005-2015)*
}

\author{
Determinants of inflation in the Democratic Republic of the Congo: \\ an econometric study (2005-2015)
}

Christian Ndege Kobunda, Ricardo Ramalhete Moreira e Edson Zambon Monte**

\begin{abstract}
Resumo: Em muitos países emergentes e subdesenvolvidos, os choques de oferta provaram ser os maiores causadores da inflação a partir dos anos 2000 (DE GREGORIO, 2012). As mudanças de preços das commodities e da taxa de câmbio nominal mostraramse relevantes para o entendimento da dinâmica inflacionária destes países. Assim, este trabalho teve por objetivo analisar os determinantes da inflação na República Democrática do Congo (RDC), entre janeiro de 2005 e dezembro de 2015, utilizando o modelo dos Vetores Autorregressivos (VAR), por meio das funções de resposta ao impulso (FRis) e da decomposição da variância do erro de previsão (DV). Os resultados mostraram que o pass-through do câmbio foi a variável com maiores impactos, positivos e significativos, sobre a inflação, e que a taxa de juros se revelou um instrumento estatisticamente insignificante para combater a inflação no país.
\end{abstract}

Palavras-chave: República Democrática do Congo; Taxa de Inflação; Taxa de Câmbio; Taxa de Juros; Commodities; VAR

\begin{abstract}
In many emerging and underdeveloped countries, supply shocks have proved to be the main drivers of inflation since the 2000s. Changes in commodity prices and in the nominal exchange rate have been relevant to understanding inflationary dynamics. Thus, this paper aimed to analyze the determinants of inflation in the Democratic Republic of Congo (DRC), from January 2005 to December 2015, using the Vector Autoregressive model (VAR), through impulse response functions (FRis) and variance decomposition or forecast error variance decomposition (VD). The results showed that the pass-through was the variable with the strongest positive and
\end{abstract}

\footnotetext{
* Submissão: 14/10/2019 | Aprovação: 11/04/2020 | DOI: 10.5380/re.v42i77.69673

** Respectivamente: (1) Mestre em Economia pela Universidade Federal de Espírito Santo (UFES) e bolsista no Instituto Jones dos Santos Neves (IJSN) | E-mail:christianndege@yahoo.fr | ORCID: 00000002-7523-9767 | (2) Professor do Departamento de Economia, do Programa de Pós-Graduação em Economia (PPGEco) e membro do Grupo de Pesquisa em Econometria (GPE) da Universidade Federal do Espírito Santo (UFES) | E-mail: ricardo.moreira@ufes.br | ORCID: 0000-0002-1905-4872 | (3) Professor do Departamento de Economia, do Programa de Pós-Graduação em Economia (PPGEco) e membro do Grupo de Pesquisa em Econometria (GPE) da Universidade Federal do Espírito Santo (UFES) | E-mail: edsonzambon@yahoo.com.br | ORCID: 0000-0002-6878-5428
} 
significant impacts on inflation, and that the interest rate was a statistically nonsignificant instrument to combat inflation in the country.

Keywords: Democratic Republic of Congo; Inflation Rate; Exchange Rate; Interest Rate; Commodities; VAR

JEL: E31; E52; C32 


\section{Introdução}

A República Democrática do Congo tem histórico de inflação alta desde sua independência, em junho de 1960. A média inflacionária entre 1970 e 1990 foi superior a 50\%, ultrapassando 256\% em 1990. Em toda a década de 1990 e no início dos anos 2000, a economia da RDC acumulou vários desequilíbrios macroeconômicos, tais como: degradação acentuada das atividades de produção, a deterioração das finanças públicas, a piora da inflação, instabilidades das reservas cambias e o acúmulo de serviços de dívidas. Este período foi de declínio da economia e da sociedade congolesas. A longa transição política não só marcou a instabilidade institucional, mas acima de tudo, os males sociais (conflitos étnicos em 1991 e 1992, greves, protestos populares, etc.) e econômicos (hiperinflação, ruptura das cooperações multilaterais e bilaterais). Embora estes desequilíbrios tenham afetado significativamente as contas do país, o Estado continuou financiando a emissão de moeda nacional levando a economia a uma espiral de hiperinflação (984\% a.a.), à maxidesvalorização da moeda nacional e à dolarização da economia (Lendele e Kamanda, 2005; Fisher et al., 2013). Essa prática era comum em muitos países africanos como, por exemplo, a Tanzânia (Solomon e De Wet, 2004).

Durante esses anos começou então o processo de democratização, em meio à ruptura com o FMI e à desintegração do Estado e da economia nacional. Houve desordem geral das finanças públicas, evidenciada pelos déficits insustentáveis do Tesouro.

Se grandes déficits públicos estão associados à alta inflação, como ocorreu com a RDC até meados da década de 1990, a forma como esses déficits são financiados pode ter implicações para o movimento futuro da inflação. Até o começo da década de 1990, os déficits orçamentários nesses países africanos eram financiados majoritariamente através de empréstimos externos, que cobravam altas taxas de juros (em função da instabilidade político-econômica persistente, como é o caso da RDC). Daí decorre a importância da união econômica. Quando um país africano entra num bloco econômico, ele passa a gozar de vários benefícios que vão além das vantagens do livre comércio tradicionais, mas também incluem acesso aos empréstimos a juros mais baixos (existem instituições financeiras próprias como, por exemplo, o Banco Africano para o Desenvolvimento, entre outras). 
Na década de 2000, por sua vez, houve um esforço grande do governo para melhorar seu quadro macroeconômico, apoiado por programas de assistência de instituições financeiras internacionais a partir de 2002, sobretudo, com o retorno das relações com o FMI e o Banco Mundial, permitindo a criação do Programa Intermediário Reforçado (PIR), que visava restaurar fundamentos institucionais para lançar as bases para a reconstrução da economia nacional, lutando contra a hiperinflação, a hiperdesvalorização da moeda e a prática de financiar o déficit orçamentário via emissão monetária. Assistiu-se então à queda significativa da taxa de inflação, que saiu de três para dois, e em sequência para apenas um dígito (Lendele e Kamanda, 2005).

Segundo Moreira (2012; 2014), os choques de oferta são os maiores causadores de inflação nos países exportadores de commodities. Entende-se que na RDC, sendo ela uma pequena economia aberta dependente das exportações (e importações) de commodities, as variações de tais preços e as oscilações da taxa de câmbio são refletidas na dinâmica da inflação ao consumidor, assim como ocorre em vários países dependentes de tais bens.

A RDC é a $94^{\mathrm{a}}$ maior exportadora de commodities no mundo e a $125^{\mathrm{a}}$ maior importadora mundial. No período entre 2005 e 2015, sua balança comercial esteve deficitária (OEC, 2017). Um dos efeitos adicionais dos ciclos de preços de commodities para a RDC parece ser sobre o orçamento do governo. Em muitos países exportadores de commodities uma grande parcela da receita do governo depende delas. Assim, os choques nos preços das commodities tendem a causar grandes flutuações nas receitas fiscais e, correspondentemente, mudanças muito grandes nos gastos do governo. De fato, uma análise empírica dos países exportadores de petróleo sugere que os choques nos preços do petróleo afetam o crescimento (FMI, 2015).

Em estudo empírico realizado para o Brasil, Pereira e Carvalho (2000) mostraram que uma depreciação cambial gera uma pressão sobre a estrutura de custos por meio de encadeamentos intersetoriais capazes de elevar a inflação ao consumidor. Por conseguinte, o aumento da inflação derivada dos choques de oferta e a alta dos custos são combatidos de modos diferentes em cada país. $\mathrm{Na}$ RDC, por exemplo, o Banco Central do Congo (BCC) usa a taxa de juros como instrumento de regularização da liquidez. Pode-se supor que o BCC segue um comportamento contracíclico no que diz respeito ao controle da inflação, ou seja, 
que há uma política monetária condizente com o princípio de Taylor. Contudo, tal hipótese também ainda carece de melhor verificação empírica.

No intuito de obter respostas sobre o que determina de fato a inflação na $\mathrm{RDC}$, assim como evidências acerca da natureza da política monetária do BCC, o presente trabalho optou por usar a metodologia de Vetores Autorregressivos (VAR), que possibilita entender as inter-relações dinâmicas entre séries temporais e de modo irrestrito, ou seja, sem a imposição de restrições estruturais nos parâmetros que regem as mencionadas relações. Avaliou-se o tempo de reação aos choques nas variáveis, a duração e a direção das repostas, especialmente as semelhanças e diferenças das respostas da inflação frente a choques nas variáveis macroeconômicas.

Foram utilizados dados mensais da RDC abrangendo o período de janeiro de 2005 a dezembro de 2015, adequando-se à disponibilidade restrita de dados, incluindo-se o índice geral de preços internacionais de commodities (calculado pelo FMI), a taxa nominal de câmbio e a taxa básica de juros, ambas fornecidas pelo BCC, e a taxa de inflação (calculado pelo INS e BCC). Vale mencionar que este trabalho contribui para a literatura macroeconômica aplicada, dada a escassez de estudos sobre o tema aplicados à República Democrática do Congo.

Além desta introdução, o presente artigo está organizado da seguinte maneira: na seção 2 serão apresentados o escopo teórico e os resultados empíricos encontrados na literatura, dando-se ênfase ao impacto de um choque nos preços de commodities e ao efeito do repasse (pass-through) do câmbio para a inflação. Já na seção 3, apresentam-se os procedimentos empíricos. Por fỉm, a seção 4 apresenta a análise dos resultados e discussões, seguidas pelas considerações finais e referências utilizadas.

\section{Revisão da literatura}

\subsection{Breve escopo teórico}

As questões referentes aos choques de oferta, como, por exemplo, as variações das cotações internacionais das commodities e os movimentos cambiais, são pontos de grande interesse nos estudos sobre política monetária, dada a importância dessas variáveis na dinâmica da inflação em determinadas economias. Conforme Ca’Zorzi, Hahn e Sánchez (2007) e Chen (2008), compreender o mecanismo de repasse dessas variáveis permite que a autoridade monetária 
responda tempestivamente e na intensidade adequada aos eventuais choques, minimizando seu impacto inflacionário e o custo econômico da atuação do Banco Central (MELO, 2010). Autores como Cashin, Liang e Mcdermott, (2000), Anzuini et al. (2003), Svenson (2005), Böwer, Geis e Winkler (2007), Rosengren (2011), Gelos e Ustyugova (2012), De Gregorio, (2012), Arezki et al., (2013) e Moreira (2012a; 2014) estudaram os efeitos, mecanismos e a transmissão dos choques dos preços das commodities sobre a inflação.

Numa economia exportadora-líquida de commodities a expectativa é de que um aumento das cotações de tais bens no mercado internacional gere uma valorização cambial, dada a maior entrada de capital e de divisas (De Gregorio e Wolf, 1994; Froot e Rogoff, 1996; Fernandez, 2003; Clements e Fry, 2006; Chen, 2008). Por sua vez, deve-se atentar para o efeito pass-through do câmbio sobre a inflação ao consumidor. Segundo Couto e Fraga (2015), o pass-through do câmbio é definido como a variação percentual dos preços domésticos frente à variação de $1 \%$ na taxa de câmbio ${ }^{1}$. Caso a lei dos preços únicos e a paridade de poder de compra fossem válidas, o grau de pass-through seria igual à unidade, ou seja, completo (Campa e Goldberg, 2002). Todavia, ele pode ser nulo, se as variações cambiais não são repassadas aos preços devido a restrições no processo de remarcação de preços pelas empresas. De outro modo, o pass-through pode encontrar-se entre zero e um e, neste caso, apenas parte da variação cambial é repassada aos preços, resultando em um pass-through incompleto (Colbano, 2006), o qual pode ser considerado o caso geral de análise. O repasse cambial pode ser tanto no âmbito macro (país ou grupo de países), quanto no microeconômico (setores de atividade) (Goldfajn e Werlang, 2000; Schmidt-Hebbel e Tapia, 2002; Taylor, 2000).

\subsection{Evidências empíricas}

Cashin, Céspedes e Sahay (2004), por meio de testes de cointegração e da metodologia de correção de erro proposta por Engle e Granger (1987), testaram a relação de longo prazo e a direção de causalidade entre os preços das commodities e as taxas de câmbio de 58 países exportadores de commodities, entre 1980 e 2002. As evidências mostraram que variações nos preços de commodities afetaram o

\footnotetext{
${ }^{1}$ Vale mencionar que este repasse é observado na maioria dos casos em países com adoção de câmbio flutuante como da RDC.
} 
câmbio real de longo prazo de um terço da amostra, contrariando os resultados de modelos de paridade do poder de compra (PPP), segundo os quais a taxa de câmbio de longo prazo deveria ser constante.

Moreira (2012) analisou as origens da taxa de inflação ao consumidor brasileiro entre janeiro de 2005 e junho de 2011, usando a metodologia VAR, no intuito de captar medidas pelas quais a inflação ao consumidor é determinada pelos choques de oferta (preços das commodities) ou pelos choques da demanda (dinâmica da atividade econômica). Como resultado, o autor verificou que a variação da taxa de inflação ao consumidor brasileiro foi determinada pela flutuação dos preços das commodities com defasagens de tempo (no sentido de Granger), em vez de pela dinâmica da atividade doméstica. Tais evidências representariam uma situação de trade-off para o Banco Central, já que, sob choques de oferta, haveria uma troca da menor variabilidade da inflação por maior variabilidade do produto.

Mallick e Souza (2013), por sua vez, analisaram as relações entre política monetária, preços de commodities e pressões inflacionárias para o BRICS (Brasil, Rússia, Índia, China e África do Sul), no período entre 1990 e 2012, com o uso de técnicas econométricas, tais como VAR, BVAR (bayesiano) e PVAR (VAR em painel). Os resultados mostraram que uma contração da política monetária teve um efeito negativo sobre a produção. Também mostraram que tal contração tende a estabilizar a inflação nesses países, embora em um nível mais alto, devido à inflação impulsionada pelos choques de oferta, especificamente com o aumento nos preços de alimentos e combustíveis, enquanto produz um efeito negativo fortemente persistente sobre os preços reais das ações.

Loloh (2014) estimou o impacto do repasse dos movimentos da taxa de câmbio nos preços domésticos em Gana, durante o período de 1994 a 2012, usando um VAR recursivo. Os resultados mostram que o efeito de um choque da taxa de câmbio nominal sobre os preços domésticos é incompleto, geralmente modesto, desaparece entre 18 e 24 meses, mas seu efeito é mais sentido durante os primeiros 12 meses. No geral, o impacto de um choque da taxa de câmbio no índice de preços ao consumidor foi menor do que nos preços de bens não alimentares. Além disso, evidências foram encontradas a favor da suposição de Taylor de que o grau de repasse da taxa de câmbio está positivamente correlacionado com o nível de inflação. 
Lariau, El Said e Takebe (2016) estudaram o repasse em Angola e na Nigéria usando teste de cointegração e VEC. Embora ambos os países compartilhem a mesma dependência das exportações de petróleo, os resultados encontrados diferem. Para Angola, o grau de repasse da taxa de câmbio aos preços domésticos foi alto no longo prazo. Nos anos 2000, este repasse se reduziu, refletindo o processo de desdolarização da economia. Já para a Nigéria, por meio do VAR, não houve uma relação estável de longo prazo entre a taxa de câmbio e os preços, isto é, as mudanças na taxa de câmbio não têm um efeito pass-through significativo sobre a inflação. O período de análise foi de maio de 2005 a abril de 2015 para Angola, para isolar a guerra e os efeitos imediatos do pós-guerra, enquanto para a Nigéria, foi entre janeiro de 1999 e abril de 2015 . O período da Nigéria corresponde ao restabelecimento da democracia e coincide com a mudança de um regime de taxa de câmbio fixa para flutuante.

Menga (2010) estudou o grau de repasse da taxa de câmbio sobre a inflação no período de 2002 a 2007 para a RDC. A metodologia de Vetores Auto Regressivos foi escolhida para destacar a relação do índice de preços ao consumidor (IPC) e do índice de preços da gasolina frente às mudanças da taxa nominal de câmbio. Os resultados da análise empírica mostraram que as mudanças da taxa de câmbio levam a uma resposta positiva e rápida do índice de preços ao consumidor e do índice de preços da gasolina.

Por sua vez, para a RDC, Fisher, Lundgren e Jahjah (2013) estimaram o vetor de cointegração usando a abordagem de Johansen (1988). Os autores mostraram que existe uma relação entre três variáveis endógenas no período entre 2002 e 2013: o IPC, a base monetária e a taxa de câmbio nominal. No longo prazo, o nível de preço é essencialmente determinado pela taxa de câmbio nominal - o que não surpreende, dada a alta dolarização da economia da RDC - e pela base monetária. A elasticidade dos preços no longo prazo para a taxa de câmbio nominal e para a base monetária eram significativas e, respectivamente, 0,80 e 0,31. O efeito colateral da dolarização constitui uma restrição importante para a condução da política monetária no Congo. Já a dinâmica de curto prazo do modelo demonstrou que, apesar da alta ponderação de alimentos na cesta do IPC, não houve um impacto relevante dos produtos alimentares sobre a inflação. Em contraste, o preço mundial do petróleo teve um forte impacto sobre a inflação na RDC. 
Mabulango e Boboy (2016) estudaram o mecanismo de transmissão da política monetária na República Democrática do Congo usando dados mensais para o período de janeiro de 2003 a dezembro de 2015. Seguindo o trabalho de Sims e Zhao (1998), Brandt e Freedman (2006) e Koop e Korobilis (2010), um modelo VAR do tipo bayesiano foi usado para estudar a dinâmica das respostas de curto prazo do produto e da inflação aos choques de taxa de juros e taxa de câmbio. Os autores verificaram que o mecanismo de transmissão pelo canal de taxa de juros continua fraco devido à natureza do sistema financeiro nacional e à presença de um setor informal amplo e segmentado. Assim, segundo os autores, para fortalecer o mecanismo de transmissão por meio do canal de taxas de juros, os formuladores de políticas são incentivados, por exemplo, a adotar medidas para fortalecer a supervisão bancária e a supervisão micro e macroprudencial. Além disto, a reforma dos setores de seguros, previdência e a consolidação do setor de microfinanças devem ser incentivadas, pois facilitariam a expansão dos serviços financeiros e ajudariam a atrair investidores no longo prazo.

Por fim, Pinshi e Sungani (2018) testaram a relação entre as variações da taxa de câmbio e os preços domésticos na RDC, mais particularmente para medir o grau de repasse da taxa de câmbio para a inflação no período de janeiro de 2002 a março de 2017. Por meio do teste de cointegração e usando um Vetor de Correção de Erros (VEC), os autores mostraram que o pass-through é relativamente elevado no Congo e que $1 \%$ de depreciação cambial elevava a inflação em $0,38 \%$ no curto prazo. Já no longo prazo, a depreciação de câmbio em $1 \%$ elevava a inflação em $1,66 \%$.

\section{Procedimentos empíricos}

\subsection{Modelo de Vetores Autoregressivos e estratégia metodológica}

A metodologia econométrica VAR possibilita a análise de modelos econômicos completos. Pode-se expressar um modelo VAR de ordem $\mathrm{p}$ em função de um vetor com n variáveis endógenas, $X_{t}$, sendo que estas se conectam por meio de uma matriz $A$, da seguinte forma:

$$
A X_{t}=B_{0}+\Sigma_{\mathrm{i}=1}^{p} B_{i} X_{t-i}+B \varepsilon_{t}
$$


em que $A$ é uma matriz $n \times n$ que define as restrições contemporâneas entre as variáveis que constituem o vetor $n \times 1, X_{t} ; B_{0}$ é um vetor de constantes $n \times 1$; $B_{i}$ são matrizes $n \times n$; $B$ é uma matriz diagonal $n \times n$ de desvios-padrão; e, $\varepsilon_{t}$ é um vetor $n \times 1$ de perturbações aleatórias não correlacionadas entre si contemporânea ou temporalmente, isto é:

$$
\varepsilon_{t} \sim \text { i.i.d }\left(0 ; I_{n}\right)
$$

em que 0 é o vetor nulo e $I$, a matriz identidade.

A equação 1 expressa as relações entre as variáveis endógenas, geralmente advindas de um modelo econômico teoricamente estruturado, e é denominada de forma estrutural. No entanto, devido à endogeneidade e problemas de identificação das variáveis do VAR, o modelo é normalmente estimado em sua forma reduzida, dada por:

$$
X_{t}=A^{-1} B_{0}+\sum_{\mathrm{i}=1}^{p} A^{-1} B_{i} X_{t-i}+A^{-1} B \varepsilon_{t}=\Phi_{0}+\sum_{\mathrm{i}=1}^{p} \Phi_{i} X_{t-i}+e_{t}
$$

em que $\Phi_{i} \equiv A^{-1} B_{i}, \quad i=0,1, \ldots, p, e B \varepsilon_{t} \equiv \mathrm{A} e_{\mathrm{t}}$.

A metodologia VAR é simplesmente uma forma reduzida de sobreposições de algumas regressões do modelo simultâneo (HAMILTON, 1994, p. 326-327). Ademais, uma das virtudes do VAR é a clareza que proporciona na tomada de decisão de quais variáveis contemporâneas são exógenas. A escolha do modelo VAR é também pela sua flexibilidade, facilidade de estimar e geralmente acompanhado por bom ajuste aos dados macroeconômicos (JUSELIUS, 2006).

A metodologia VAR pode ser estimada por meio do método de MQO, levando-se em conta, principalmente, a interação entre as variáveis do sistema considerado. Dentre as suas principais vantagens na análise econométrica estão a obtenção das funções de respostas ao impulso (FRI) e a decomposição da variância do erro de previsão (DV).

Para obtenção das FRI foram utilizados dois procedimentos: Cholesky e Generalizado para fins comparativos. Ressalta-se que se optou por dar maior ênfase à significância estatística nas análises neste artigo. Antes de estimar as funções de impulso-resposta, é fundamental identificar o ordenamento de 
causalidade contemporânea de Cholesky no modelo. O procedimento de ortogonalização dos resíduos do VAR, com base na chamada "decomposição de Cholesky", impõe uma estrutura recursiva à matriz de relações contemporâneas entre as variáveis do modelo - de modo que a primeira variável não seja afetada contemporaneamente por nenhuma das demais, a segunda seja afetada apenas pela primeira, a terceira seja afetada pelas primeiras duas, e assim por diante (Cavalcanti, 2010).

A utilização do procedimento Generalizado, por sua vez foi introduzido originalmente na macroeconometria por ser insensível ao ordenamento de Cholesky. Diferentemente do que ocorre na tradicional decomposição de Cholesky, que se utiliza da "hipótese da ortogonalidade", o método de impulso-resposta generalizado não varia caso ocorra reordenamento das variáveis no VAR (Lütkepohl, 2006; Koop, Pesaran e Potter, 1996; Pesaran e Shin, 1998). Conforme Edwing (2003), o método generalizado apresenta duas vantagens principais: a) os resultados da função resposta ao impulso generalizado apresentam maior robustez do que o método ortogonalizado (Cholesky); e b) uma vez que a ortogonalidade não é imposta, a FRI generalizada possibilita uma interpretação com maior acurácia do efeito inicial de um choque de variável sobre as demais variáveis.

A decomposição dos erros de previsão (DV) mostra o comportamento dinâmico apresentado pelas variáveis econômicas. Mais especificamente, este instrumental permite separar a variância do erro de previsão para cada variável em componentes que podem ser atribuídos pelas demais variáveis endógenas isoladamente, ou seja, revela em termos porcentuais qual o efeito que um choque não antecipado sobre determinada variável tem sobre as demais variáveis pertencentes ao sistema. No caso da DV, a questão referente à ordenação das variáveis é de suma importância. Utilizou-se o ordenamento de Cholesky: D(COMM), D(CAMBIO), INF, D(JUROS).

\subsection{Apresentação das variáveis}

Este estudo compreendeu estimativas para o período entre janeiro de 2005 a dezembro de $2015^{2}$, utilizando dados mensais e perfazendo um total de 132

\footnotetext{
${ }^{2}$ A escolha do período foi em função da disponibilidade restrita dos dados. Na construção do banco de dados para a pesquisa, observou-se a falta de dados mensais para o período antes de 2005 . A maioria dos estudos realizados que cobriram os anos 90 tem frequência anual, incorrendo em baixo número de observações, dado que a independência da RDC foi em 1960. Portanto, optou-se por usar dados mensais,
} 
observações. Os dados foram coletados no Banco Central do Congo (BCC), no Instituto Nacional de Estatísticas (INS) e no Fundo Monetário Internacional (FMI).

Tabela 1 - Variáveis, unidades, siglas e fontes

\begin{tabular}{cccc}
\hline Variáveis & Unidade & Sigla & Fonte \\
\hline Inflação & Porcentagem & INF & BCC/INS \\
Câmbio & CDF/U\$D & CAMBIO & BCC/INS \\
Juros & Porcentagem & JUROS & BCC/INS \\
Commodities & Índice & COMM & FMI \\
\hline
\end{tabular}

Fonte: Elaboração própria.

A Tabela 1 traz a descrição completa de todas as variáveis utilizadas. A taxa de inflação (INF) é o aumento no nível de preços ao consumidor em variação mensal. No que se refere ao câmbio nominal (CAMBIO), mede-se o valor do franco congolês (CDF) em relação ao dólar americano (US\$), ou seja, a relação CDF/US\$. A taxa de juros (JUROS), expressa em porcentagem, mês a mês, expressa a taxa básica de juros usada pelo BCC para fins de política monetária. Já o Índice Geral de Commodities (COMM) tem por objetivo medir os preços das commodities no mercado internacional.

\subsection{Gráficos das variáveis e estatísticas descritivas}

Na Figura 1 são observadas as trajetórias temporais das variáveis COMM, CAMBIO, INF e JUROS, considerando o período como um todo (2005-2015). A Tabela 2 apresenta as estatísticas descritivas básicas em nível. Constata-se na Figura 1 que, antes de 2012, a variável de principal interesse (inflação) teve maior variância, apresentando valores, por exemplo, em junho de 2005, de -7,20\% e, em março de 2009, de 8,30\%. A partir de fevereiro de 2012, a inflação reduziu significantemente na economia do Congo. A inflação estabilizou-se então abaixo de $1 \%$ ao mês, o que vai ao encontro da estabilidade da taxa básica de juros, a qual, até 2012, também apresentou variância consideravelmente maior do que o observado nos anos seguintes.

O índice de commodities segue uma trajetória de crescimento até o último mês de 2008. Depois disso, teve uma queda por causa da crise do subprime, para

elevando o número de observações, restringindo-se ao período para o qual tais séries estão disponíveis pelos órgãos oficiais do país. 
depois voltar para a sua trajetória de crescimento. Nota-se que a taxa de câmbio teve uma tendência de crescimento até se estabilizar em 2012 devido ao aumento das reservas internacionais no Congo.

\section{Figura 1 - Evolução das variáveis (em nível) no período de} Jan/2005 a Dez/2015
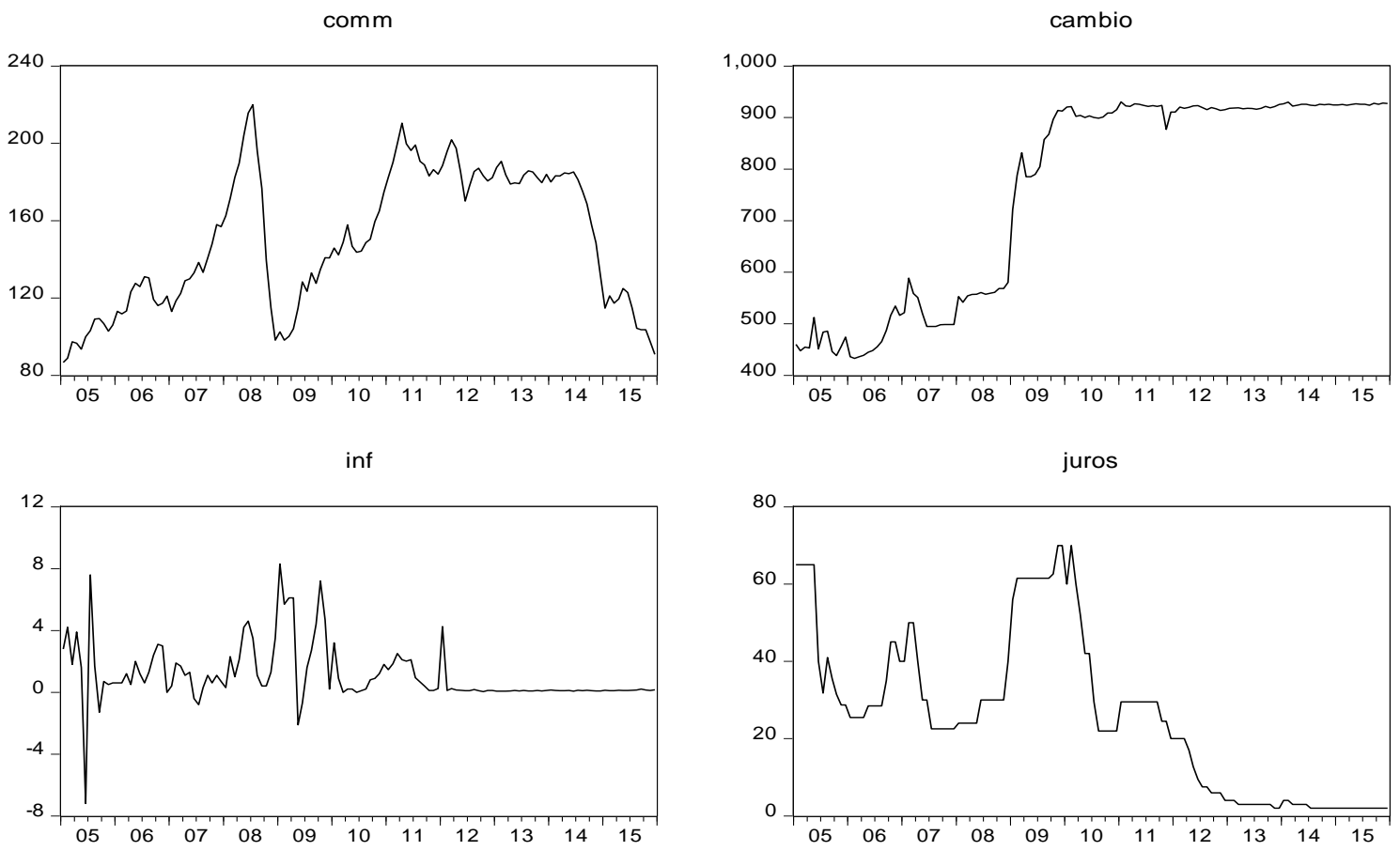

Fonte: Elaboração própria.

Em relação à Tabela 2, vê-se que a série de inflação é leptocúrtica e mostra um forte desvio da normalidade (os coeficientes de assimetria e curtose são todos diferentes daqueles da distribuição normal padrão, que são 0 e 3 , respectivamente). Percebe-se, também, uma disparidade muito grande entre o valor máximo e o mínimo, 8,30 e -7,20. Reforçando isso, cabe destacar que o teste de Jarque-Bera (JB) rejeitou a hipótese nula de normalidade ao nível de significância de 5\% para todas as variáveis. Por fim, a inflação registrou uma média de 1,09 e um desvio padrão de 1,90 . 
Tabela 2 - Estatísticas Descritivas de Jan/2005 a Jan/2015

\begin{tabular}{cccccccccc}
\hline Variável & \multirow{2}{*}{ Média } & \multirow{2}{*}{ Mediana } & Máximo & Mínimo & $\begin{array}{c}\text { Desvio- } \\
\text { padrão }\end{array}$ & $\begin{array}{c}\text { Assimet } \\
\text { ria }\end{array}$ & Curtose & $\begin{array}{c}\text { Jarque- } \\
\text { Bera }\end{array}$ & Valor-p \\
\hline INFL & 1,0971 & 0,350 & 8,300 & $-7,200$ & 1,9029 & 0,9393 & 7,8962 & 151,26 & 0,000 \\
CAM & 759,34 & 906,42 & 930,47 & 432,88 & 199,57 & $-0,567$ & 1,4588 & 20,139 & 0,000 \\
JUROS & 26,038 & 24,50 & 70,000 & 2,000 & 20,65 & 0,5429 & 2,304 & 9,1496 & 0,0103 \\
COMM & 149,71 & 147,28 & 220,03 & 86,617 & 35,369 & $-0,0116$ & 1,656 & 9,9369 & 0,0069 \\
\hline
\end{tabular}

Fonte: Elaboração própria.

\section{Resultados}

\subsection{Teste de raiz unitária}

Como um dos primeiros passos na análise de séries temporais está a verificação da ordem de integração das séries. Caso não sejam estacionárias, devese realizar algum procedimento para estacionarizá-las (em geral, aplica-se a primeira diferença nas mesmas, dado que a maioria das séries econômicas é integrada de primeira ordem).

Neste trabalho foram utilizados os seguintes testes: Augmented DickeyFuller - ADF (Dickey e Fuller, 1981), Phillips-Perron - PP (Phillips e Perron, 1988) e Kwiatkowski-Phillips-Schmidt-Shin - KPSS (Kwiatkowski et al., 1992). Na realização do teste ADF foi utilizado o critério de informação de Schwarz (SIC) com o número de defasagens escolhido de forma automática e um máximo de 12 defasagens. No caso do teste PP, adotou-se o método de estimação espectral de Barllet Kernel e o critério de seleção de defasagens automático de Newey-West Bandwidth. Para o teste KPSS também foram utilizados a estimação espectral de Barllet Kernel e o critério Newey-West Bandwidth. A Tabela 3 apresenta os resultados dos testes citados, que demonstram que é necessário aplicar a primeira diferença nas variáveis CAMBIO, JUROS e COMM, pois somente a variável INF é estacionária em nível (Tabela 4).

Tabela 3 - Teste de raiz unitária em nível

\begin{tabular}{cccccccc}
\hline VARIÁVEL & ADF & $\mathrm{K}$ & $\mathrm{PP}$ & $\mathrm{K}$ & $\mathrm{KPSS}$ & $\mathrm{K}$ & \\
\hline INF & $-3,011^{* *}$ & 5 & $7,765^{* * *}$ & 1 & $0,764^{\mathrm{ns}}$ & 4 & $\mathrm{cc}$ \\
CAMBIO & $-1,354^{\mathrm{ns}}$ & 1 & $-1,246^{\mathrm{ns}}$ & 1 & $1,206^{* * *}$ & 9 & $\mathrm{cc}$ \\
JUROS & $-1,981^{\mathrm{ns}}$ & 1 & $-1,958^{\mathrm{ns}}$ & 6 & $0,870^{* * *}$ & 9 & $\mathrm{cc}$ \\
COMM & $-2,267^{\mathrm{ns}}$ & 1 & $-2,077^{\mathrm{ns}}$ & 6 & $0,478^{* *}$ & 5 & $\mathrm{cc}$ \\
\hline
\end{tabular}

Fonte: Elaboração própria. 
Nota: 1) *** Significativo a $1 \%$, ** Significativo a $5 \%$, * Significativo a $10 \%$, (ns) Não significativo a $10 \%$; 2) $\mathrm{K}=$ número de defasagens de cada teste para cada variável; 3) (ct) representa com constante e com tendência e (cc) com constante.

\section{Tabela 4 - Testes de raiz unitária em primeira diferença}

\begin{tabular}{cccccccc}
\hline VARIÁVEL & ADF & K & PP & K & KPSS & K & \\
\hline D(CAMBIO) & $-10,51^{* * *}$ & 0 & $-10,53 * * *$ & 3 & $0,221^{\mathrm{ns}}$ & 1 & $\mathrm{cc}$ \\
D(JUROS) & $-9,028^{* * *}$ & 0 & $-9,125 * * *$ & 6 & $0,067^{*}$ & 6 & $\mathrm{cc}$ \\
D(COMM) & $-6,517^{* * *}$ & 0 & $-6,550^{* * *}$ & 2 & $0,249^{*}$ & 6 & $\mathrm{cc}$ \\
\hline
\end{tabular}

Fonte: Elaboração própria.

Nota: 1) *** Significativo a $1 \%$, ** Significativo a $5 \%$, * Significativo a $10 \%$, (ns) Não significativo a $10 \%$;2) $\mathrm{K}=$ número de defasagens de cada teste para cada variável; 3) (ct) representa com constante e com tendência e (cc) com constante; 4) $\mathrm{D}=$ primeira diferença.

O próximo passo foi determinar o número apropriado de defasagens do modelo VAR. Vários critérios de informação foram utilizados para isso: os critérios da razão de verossimilhança (LR), do erro de previsão final (FPE), de Akaike (AIC), de Schwarz (SC) e de Hannan-Quinn (HQ). Esses critérios objetivam a parcimônia dos modelos, impondo penalidades pelo número de regressores utilizados. A escolha das defasagens foi condicionada à ausência de correlação serial dos resíduos, verificada por meio da estatística LM $^{3}$ (conhecido como teste Breusch-Godfrey). A defasagem ótima é sempre aquela para a qual o critério apresenta o menor valor, como mostra a Tabela 5. Os critérios SC e HQ sugeriram a utilização de uma defasagem para o modelo. Já os critérios FPE e AIC indicaram a adoção de duas defasagens. No entanto, optou-se por trabalhar com três defasagens, conforme o critério LR, uma vez que os modelos com uma e duas defasagens apresentaram autocorrelação dos resíduos.

Tabela 5 - Determinação do número de defasagens do modelo VAR

\begin{tabular}{ccccccc}
\hline Lag & LogL & LR & FPE & AIC & SC & HQ \\
\hline 0 & -1.570 .446 & NA & 838769.9 & 24.99120 & 25.08124 & 25.02778 \\
1 & -1.516 .705 & 103.2152 & 460840.6 & 24.39215 & $24.84235^{*}$ & $24.57505^{*}$ \\
2 & -1.492 .139 & 45.62242 & $402579.8^{*}$ & $24.25618^{*}$ & 25.06655 & 24.58541 \\
3 & -1.476 .842 & $27.43764^{*}$ & 407900.6 & 24.26734 & 25.43787 & 24.74289 \\
4 & -1.463 .637 & 22.84748 & 427971.2 & 24.31170 & 25.84239 & 24.93357 \\
5 & -1.452 .275 & 18.93674 & 463408.1 & 24.38532 & 26.27617 & 25.15351 \\
\hline
\end{tabular}

Fonte: Elaboração própria.

\footnotetext{
${ }^{3}$ Esse teste é feito com base na hipótese nula de ausência de autocorrelação até a defasagem especificada.
} 
Ainda, em relação à adequação dos modelos, foram realizados os testes: a) análise do padrão das raízes do polinômio estimado; e b) teste de normalidade dos resíduos de Jarque-Bera (JB). O VAR(3) apresentou todas as raízes do polinômio característicos dentro do círculo ${ }^{4}$ (Figura 2). Quanto ao teste de normalidade dos resíduos, o teste de Jarque-Bera rejeitou a hipótese nula de que os resíduos são normais. Entretanto, uma vez que não há possibilidade de elevar o tamanho da amostra, as análises foram mantidas. Destaca-se que Oreiro et al. (2006) salientam que este procedimento é comum em alguns trabalhos, como em Grôppo (2004) e Camuri (2005).

\section{Figura 2 - Inverso das raízes unitárias do polinômio até VAR(3)}

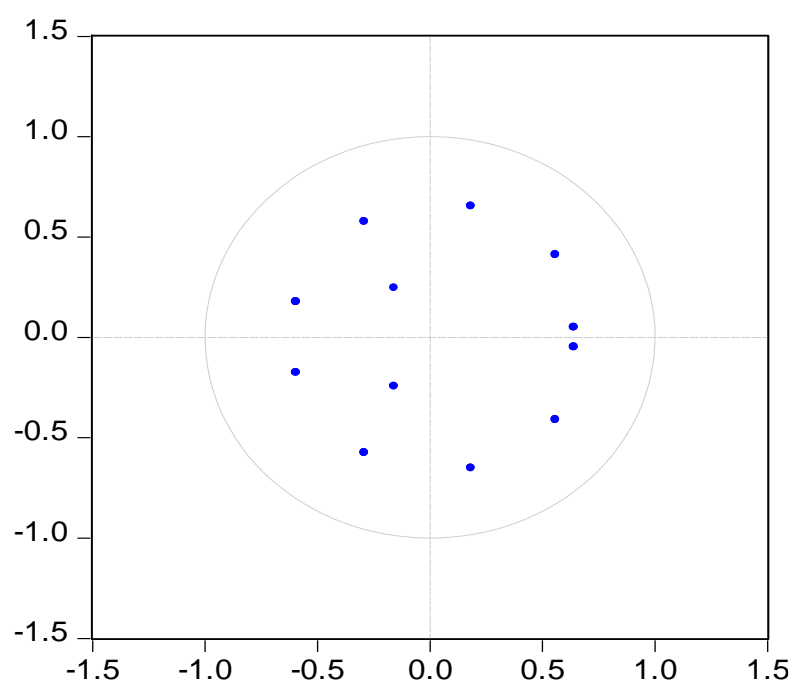

Fonte: Elaboração própria.

De acordo com o teste Bloco-causalidade de Granger de exogeneidade (Tabela 6), verificaram-se as relações causalidade bidirecional e unidirecional entre as variáveis, no sentido "Granger causa". Para cada equação do modelo VAR, pela estatística Wald, testa-se a significância de cada uma das outras variáveis endógenas desfasadas na equação. Em relação à variável de maior interesse INF, percebe-se que existe uma relação bidirecional com a variável D(CAMBIO), dando o primeiro sinal da importância do câmbio sobre a dinâmica inflacionária no Congo. Também pode-se notar a causalidade bidirecional entre as

\footnotetext{
${ }^{4} \mathrm{O}$ modelo selecionado deve apresentar todas as raízes do polinômio dentro do círculo unitário para ser considerado estável (Lütkepohl, 2006).
} 
variáveis $\mathrm{D}(\mathrm{COMM})$ e $\mathrm{D}(\mathrm{CAMBIO})$, reforçando a necessidade de entendimento dessa relação para a inflação. Para o sentido "Granger causa" unidirecional, não houve efeito de qualquer variável sobre a inflação no período. No entanto, nota-se que a variável INF causa $\mathrm{D}(\mathrm{JUROS})$ e $\mathrm{D}(\mathrm{COMM})$.

Tabela 6 - Bloco-causalidade de Granger

\begin{tabular}{ccccc}
\hline \multicolumn{5}{c}{ Variável dependente } \\
\hline Não causa & D(COMM) & D(CAMBIO) & INF & D(JUROS $)$ \\
D(COMM) & - & $11,184^{* * *}$ & $1,4545^{\text {ns }}$ & $5,2458^{\text {ns }}$ \\
D(CAMBIO) & $7,7613^{* *}$ & - & $9,6276^{* *}$ & $2,8581^{\text {ns }}$ \\
INF & $1,9013^{*}$ & $8,3414^{* *}$ & - & $23,987^{* * *}$ \\
D(JUROS $)$ & $5,6463^{n s}$ & $3,4607^{\text {ns }}$ & $0,8796^{\text {ns }}$ & - \\
Todos & $12,9874^{\text {ns }}$ & $25,268^{* * *}$ & $18,925^{* *}$ & $32,987^{* * *}$ \\
\hline
\end{tabular}

Fonte: Elaboração própria a partir dos dados da pesquisa.

Nota: 1) *** Significativo a $1 \%, * *$ Significativo a $5 \%$, * Significativo a $10 \%$, (ns) Não significativo a $10 \%$; e,2) "Todos" indica o teste de causalidade para o conjunto de todas as variáveis independentes.

Finalizada a etapa de identificação e estimação do VAR, foram então analisadas as funções impulso-resposta. $\mathrm{Na}$ Figura 3, apresenta-se $\mathrm{o}$ comportamento da inflação frente aos choques em outras variáveis num período de 12 meses. Vale reiterar que a ordenação de Cholesky escolhida foi: D(COMM), D(CAMBIO), INF, D(JUROS). Justifica-se esta ordenação porque as commodities estão totalmente fora do controle do governo e afetam o câmbio via entrada ou saída de dólares pelo comércio exterior. O câmbio, por sua vez, gera efeito na inflação, e força a política monetária a se ajustar por meio da taxa de juros.

Com base na resposta a impulso da Figura 3-A, verifica-se que a inflação responde positivamente ao choque da variação de preço das commodities no primeiro e segundo meses. Entretanto, este resultado não apresenta significância estatística. Conforme a literatura estudada, é esperado que um aumento no preço das commodities eleve os preços ao consumidor no mercado local e, consequentemente, que haja inflação doméstica. Além disso, no que tange à análise, segundo Curatola de Melo (2013), os preços das commodities podem ser caracterizados como um dos primeiros indicadores de alerta de inflação. 
Figura 3 - Função de resposta ao impulso considerando os efeitos das demais variáveis sobre a INF, com decomposição de Cholesky
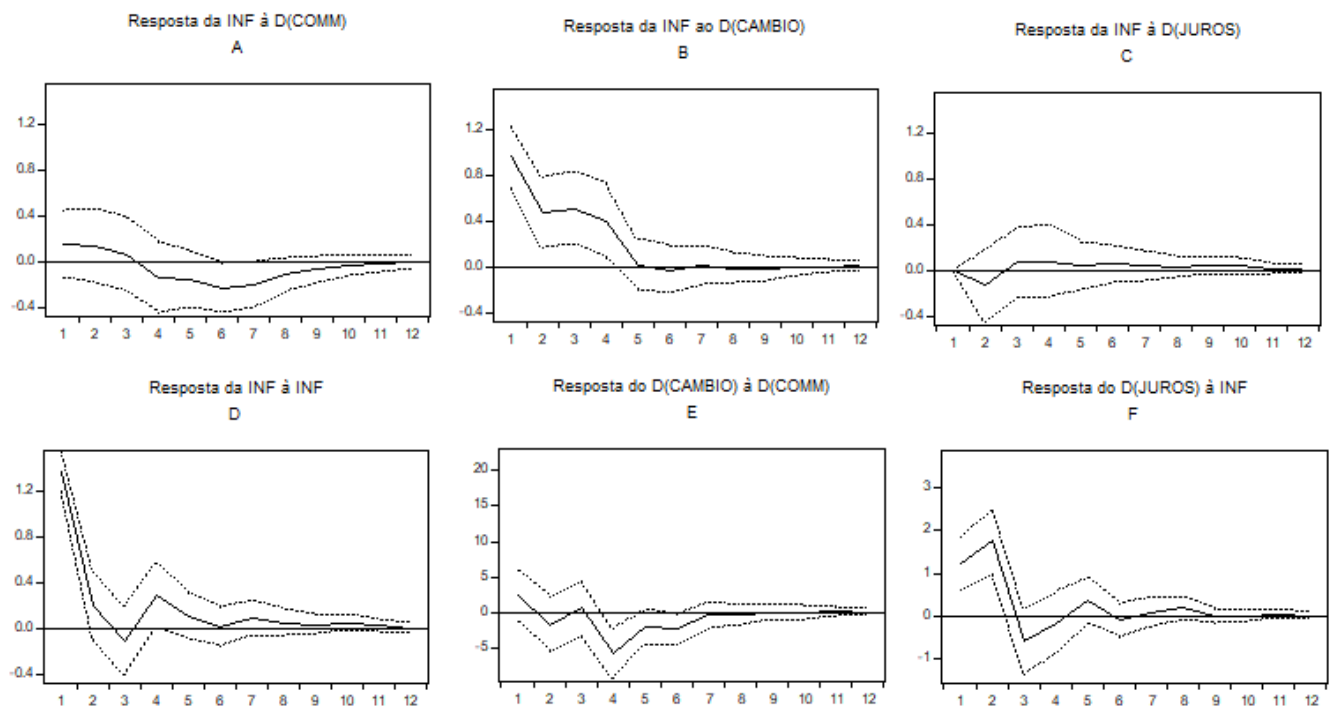

Fonte: Elaboração própria.

Na Figura 3-B, em conformidade com o teste de Bloco-causalidade de Granger de exogeneidade, o pass-through do câmbio sobre a inflação é estatisticamente significativo. Observa-se que um choque na taxa de câmbio (desvalorização cambial) causa aumento da inflação nos quatro primeiros meses e os efeitos do choque inicial somente desaparecem no quinto mês. Assim, ficam corroborados os resultados obtidos por Menga (2010), Mabulango e Boboy (2016) e Pinshi e Sungani (2018), que revelaram que a inflação congolesa é explicada, na sua maior parte, pelo câmbio.

Embora a hipótese teórica seja de uma resposta negativa da inflação à elevação da taxa de juros, na prática isso nem sempre ocorre devido a diversos fatores, como: existência de uma inércia inflacionária, ajuste insuficiente na taxa nominal de juros, falta de credibilidade da política monetária e/ou restrições fiscais severas. Conforme Fisher et al. (2013), o canal da taxa de juros continua fraco devido à natureza do sistema financeiro no Congo e à presença de um setor informal amplo e segmentado. Com base na Figura 3-C, verifica-se que a inflação responde negativamente ao choque na taxa de juros somente no segundo mês. A partir do mês seguinte, a inflação torna-se positiva para o resto do período. Todavia, este resultado não apresenta significância estatística. Vale mencionar que, mesmo não havendo resultado esperado para o período como um todo, o 
Banco Central reagiu rapidamente aos choques da inflação aumentando a taxa de juros logo no primeiro e segundo meses, como mostra a Figura 3-F (resposta com significância estatística).

A Figura 3-D demonstra que a inflação é explicada por choques nela mesma, com significância estatística. Este processo é chamado de "inércia" ou "persistência inflacionária". Na relação entre o câmbio e as commodities, que se observa na Figura 3-E, por sua vez, nota-se que o câmbio valorizou-se em consequência do choque nos preços das commodities. Em outras palavras, a elevação do preço das commodities gerou resposta positiva do câmbio no primeiro mês, mas logo no segundo mês o câmbio valorizou-se. Em termos estatísticos, somente no quarto mês aparece a significância. Tal resultado é esperado pela literatura relacionada, como foi mencionado no escopo teórico.

A Figura 4 mostra a FRI generalizada que teve por objetivo testar a robustez das estimações realizadas. Deste modo, pode-se afirmar que as estimações são robustas, dado que confirmaram as estimações realizadas pelo ordenamento de Cholesky (Figura 3). É importante notar que a FRI generalizada da Figura 4-C (INF a D(JUROS)) passou a ter significância estatística, comprovando, então, a análise efetuada na Figura 3-C, assim como o resultado levantado na Seção 2 sobre a economia congolesa.

\section{Figura 4 - Função de resposta ao impulso considerando os efeitos das demais variáveis sobre a INF, com impulso-resposta generalizada}
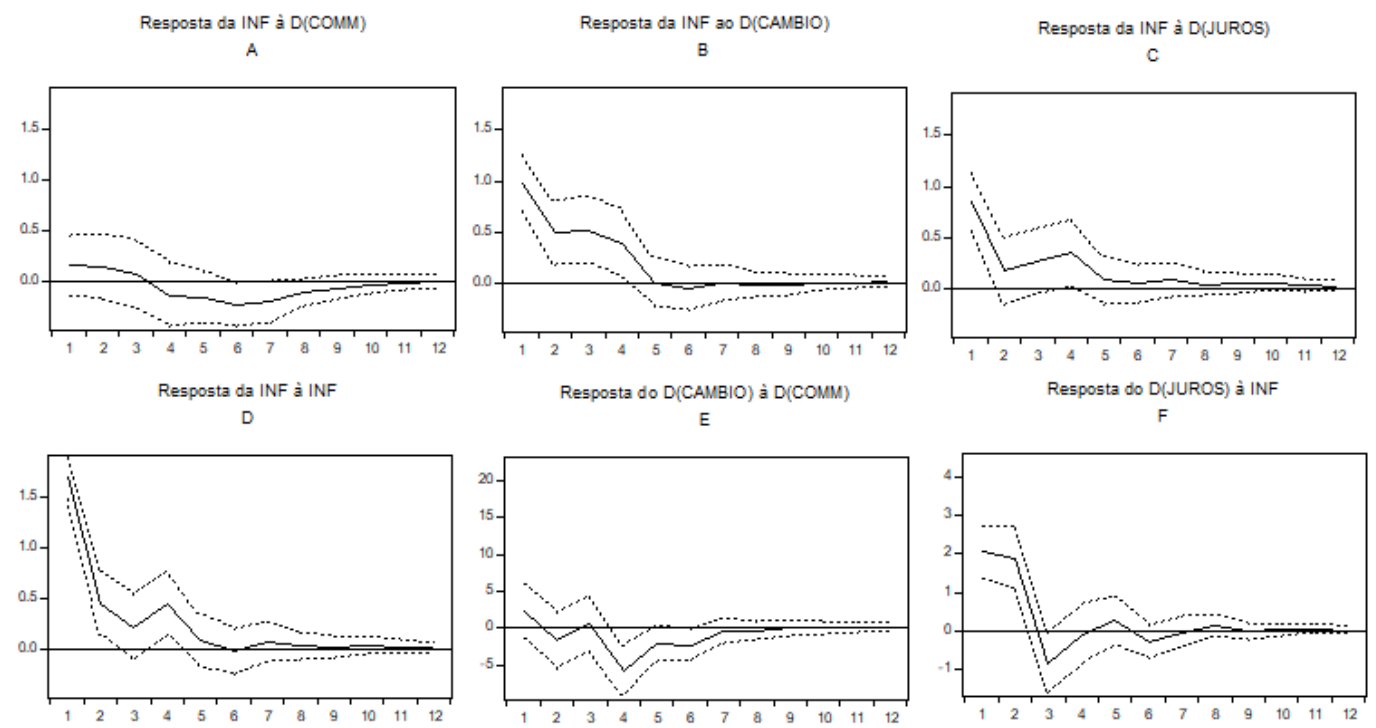

Fonte: Elaboração própria. 
Os resultados da DV para a variável INF mostram que, no primeiro mês, $66,46 \%$ do erro de previsão da inflação deve-se a ela própria e, no décimo segundo mês, cerca de 52,85\%. Ou seja, há dissipação lenta do choque inicial. Verifica-se também que, no primeiro mês de análise, a variável $\mathrm{D}(\mathrm{CAMBIO})$ teve impacto sobre o erro de previsão da inflação em aproximadamente 32,68\% e cerca de 40,73\% no décimo segundo mês. Ou seja, com o passar do tempo, o câmbio ganha espaço como determinante da inflação congolesa (Tabela 7).

Com base nessas informações, pode-se afirmar que, haja visto o histórico de inflação alta no Congo, o comportamento do câmbio vai ao encontro da previsão por parte da literatura (TAYLOR (2000), BAILLIU e FUJI (2003) e GAGNON e IHRIG (2004)), no sentido de que, num ambiente de alta inflação, o pass-through do câmbio para a inflação tende a ser persistente e elevado. Por sua vez, apesar dos níveis de explicação das variáveis $\mathrm{D}(\mathrm{COMM})$ e $\mathrm{D}(\mathrm{JUROS})$ aumentarem com o passar do tempo, eles são baixos.

Tabela 7 - Decomposição da variância da variável INF

\begin{tabular}{cccccc}
\hline Mês & Desvio-padrão & D(COMM) & D(CAMBIO) & INF & D(JUROS) \\
\hline 1 & 1,696071 & 0,850273 & 32,68197 & 66,46776 & 0,000000 \\
& & $(2,32155)$ & $(6,80193)$ & $(7,06166)$ & $(0,00000)$ \\
2 & 1,785154 & 1,376315 & 36,77069 & 61,31389 & 0,539109 \\
& & $(2,63894)$ & $(7,25468)$ & $(7,54571)$ & $(1,13177)$ \\
3 & 1,863990 & 1,387736 & 41,41448 & 56,53843 & 0,659350 \\
& & $(2,58527)$ & $(6,96557)$ & $(7,22053)$ & $(1,61164)$ \\
4 & 1,935505 & 1,796718 & 42,70311 & 54,69868 & 0,801488 \\
& & $(2,50727)$ & $(6,62744)$ & $(6,75136)$ & $(2,20803)$ \\
5 & 1,945855 & 2,463378 & 42,26332 & 54,43976 & 0,833542 \\
& & $(2,85067)$ & $(6,53072)$ & $(6,71140)$ & $(2,33340)$ \\
6 & 1,961222 & 3,883221 & 41,62409 & 53,59607 & 0,896615 \\
& & $(3,57286)$ & $(6,49260)$ & $(6,63441)$ & $(2,49453)$ \\
7 & 1,974825 & 4,915353 & 41,06113 & 53,09356 & 0,929955 \\
& & $(4,02502)$ & $(6,52422)$ & $(6,62159)$ & $(2,59787)$ \\
8 & 1,979087 & 5,233318 & 40,88611 & 52,93615 & 0,944413 \\
& & $(4,20459)$ & $(6,54309)$ & $(6,63163)$ & $(2,60637)$ \\
9 & 1,981181 & 5,328823 & 40,80653 & 52,85849 & 1,006152 \\
& & $(4,28832)$ & $(6,55161)$ & $(6,65831)$ & $(2,61332)$ \\
10 & 1,982574 & 5,347533 & 40,75004 & 52,85370 & 1,048730 \\
& & $(4,31802)$ & $(6,56484)$ & $(6,66812)$ & $(2,63630)$ \\
11 & 1,982931 & 5,349660 & 40,73619 & 52,85422 & 1,059928 \\
& & $(4,34179)$ & $(6,57044)$ & $(6,68088)$ & $(2,64599)$ \\
12 & 1,983023 & 5,349628 & 40,73548 & 52,85027 & 1,064627 \\
& & $(4,35954)$ & $(6,57371)$ & $(6,69042)$ & $(2,64980)$ \\
\hline
\end{tabular}

Fonte: Elaboração própria.

Nota: Valor em () representam os erros-padrão para 100 repetições de Monte Carlo. 


\section{Considerações finais}

O principal objetivo desse trabalho foi analisar os determinantes da inflação, mais especificamente como os choques nos preços das commodities, na taxa de câmbio e na taxa de juros têm impactado a taxa de inflação na RDC, e como e com que eficácia a política monetária do país reagiu, no período entre 2005 e 2015.

Para tanto, foi estimado um modelo de Vetores Autorregressivos (VAR) para cômputo das funções de respostas a impulsos (FRI) e da decomposição da variância do erro de previsão (DV). Dando maior ênfase à significância estatística, os resultados mostraram que os choques nos preços das commodities não mostraram ter impacto sobre a inflação nos períodos analisados. Por outro lado, os juros reagem diante de um choque na inflação ao consumidor. Isto significa que o Banco Central do Congo (BCC) foi sensível, ajustando a política monetária de acordo com a dinâmica inflacionária no país. No entanto, foram constatadas respostas positivas da inflação a choques nos juros, ou seja, o BCC aumenta os juros, mas não cria expetativas de queda da inflação, fazendo com que a inflação suba ou resista. Entende-se que isto pode ser consequência de outros fatores, tais como inércia inflacionária, ausência de credibilidade e/ou problemas fiscais, que prejudicam intensamente a atuação do BCC.

Uma das principais conclusões deste estudo foi a de que o câmbio provou ser o principal determinante da inflação no período como um todo (2005-2015). Por conseguinte, sugere-se ao Banco Central do Congo maiores esforços por ganho de credibilidade da política monetária, em conjunto com esforços de consolidação fiscal pelo Governo, assim como uma monitoração sistemática e consistente das flutuações cambiais. Uma gestão adequada das reservas internacionais por parte do BCC é indispensável, em consonância com a construção de um ambiente favorável aos investimentos estrangeiros no país, a fim de possibilitar a diversificação de setores econômicos. Em conjunto, tais medidas tendem a reduzir o efeito pass-through e a inércia inflacionária na RDC.

\section{Agradecimentos}

Os autores gostariam de agradecer ao Conselho Nacional de Desenvolvimento Científico e Tecnológico - CNPq e à Fundação de Amparo à Pesquisa e Inovação do Espírito Santo - FAPES pelo apoio financeiro. 


\section{Referências}

ANZUINI, A.; LOMBARDI, M. J.; PAGANO, P. The impact of monetary policy shocks on commodity prices. International Journal of Central Banking, v. 9, n. 3, 2013.

AREZKI, R.; HADRI, K.; LOUNGANI, P.; RAO, Y. Testing the Prebisch-Singer hypothesis since 1650: evidence from panel techniques that allow for multiple breaks. IMF, 2013. (Working paper, n. 180)

BAILLIU, J.; FUJI, E, V. Exchange rate pass-through and the inflation environment in industrialized countries: an empirical investigation, Society for Computational Economics, p. 135, 2003.

BOWER, U; GEIS, A.; WINKLER, A. Commodity price fluctuations and their impact on monetary and fiscal policies in Western and Central Africa. ECB, 2007. (Ocasional paper series, n. 60)

BUENO, R. L da S. Econometria de Séries Temporais. São Paulo: Cengage Learning, 2011.

CAMPA, J. M.; GOLDBERG, L. S. Exchange rate pass-through into import prices: a macro or micro Phenomenon. NBER, 2002. (Working paper, n. 8934)

CAMURI, P. A. Dívida pública, política fiscal e vulnerabilidade externa no Brasil. Dissertação (Mestrado). CEDEPLAR/UFMG, Belo Horizonte 2005.

CASHIN, P.; CÉSPEDES, L. F.; SAHAY, R. Commodity currencies and the real exchange rate. Journal of Development Economics, v. 75, n. 1, p. 239-268, 2004.

CASHIN, P.; LIANG, H.; MCDERMOIT, J. C. How persistent are shocks to world commodity prices? IMF Staff Papers, v. 47, n. 2, p. 177-217, 2000.

CAVALCANTI, M. A. F. H. Identificação de modelos VAR e Causalidade de Granger: Uma nota de advertência. Economia Aplicada, v. 14, n. 2, p. 251-260, 2010.

CA'ZORZI, M.; HAHN, E.; SÁNCHEZ, M. Exchange rate pass-through in emerging markets. Econstor, 2007. (Workink paper series, n. 739)

CHEN, S, S. Oil price pass-through into inflation. Energy Economics, v. 31, n. 1, p. 126-133, 2008.

CLEMENS, K, W.; FRY, R. Commodity currency and currency commodities. CAMA, 2006. (Working paper, v. 19) 
COLBANO, F. S. Preços internacionais e a taxa de câmbio: o caso brasileiro. Dissertação (mestrado). Faculdade de Economia, Administração e Contabilidade da Universidade de São Paulo. São Paulo, 2006.

COUTO, S, V, V.; FRAGA, J, G. O pass-through da taxa de câmbio para índices de preços: análise empírica para o Brasil. Revista de Economia Contemporânea, v. 18, n. 3, p. 333-356, 2014.

DE GREGORIO, J. Commodity prices, monetary policy and inflation. IMF Economic Review, v. 60, n. 4, p. 600-633, 2012.

DE GREGORIO, J.; WOLF, H. Terms of trade, productivity, and the Real Exchange Rate. National Bureau of Economic Research, 1994. (Working paper series, n. 4807)

DICKEY, D. A.; FULLER, W. A. Likelihood ratio statistics for autoregressive time series with a unit root. Econometrica, v. 49, n. 4, p. 1057-1072, 1981.

EWING, B. T. The response of the default risk premium to macroeconomic shocks. The Quarterly Review of Economics and Finance, v. 43, n. 2, p. 261-272, 2003.

FERNANDEZ, C. Y. H. Câmbio real e preços de commodities: Relação identificada através da mudança de regime cambial. Departamento de Economia, PUC-Rio. Rio de Janeiro, 2003.

FISCHER, F; LUNDGREN, C. J.; JAHJAH, S. Making monetary policy more effective: the case of the Democratic Republic of the Congo. IMF, 2013. (Working paper, n. 226)

FROOT, K.; ROGOFF, K. Perspectives on PPP and long-run real exchange rates. NBER, 1994. (Working paper, n. 4952)

GAGNON, J.; IHRIGH, J. Monetary policy and exchange rate pass-through. International Journal of Finance and Economics, v. 9, n. 4, p. 315-338, 2004.

GELOS, G.; USTYUGOVA, Y. Inflation responses to commodity price shocks: How and why do countries differ? IMF, 2012. (Working paper, n. 225)

GOLDFAJN, I.; WERLANG, S. The pass-through from depreciation to inflation: a panel study. Banco Central do Brasil, 2000. (Working paper, n. 5)

GRÔPPO, G. Causalidade das variáveis macroeconômicas sobre o IBOVESPA. Dissertação (Mestrado). ESALQ/USP, Piracicaba 2004.

HAMILTON, J, D. Time series Analysis. Princeton: Princeton University Press, 1994. 
JESELIUS, K. The cointegrated VAR model: Methodology and applications. New York: Oxford University Press, 2006.

KOOP, G.; PESARAN, M, H.; POTTER, S, M. Impulse response analysis in nonlinear multivariate models. Journal of Econometrics, v. 74, n. 1, p. 119-147, 1996.

KWIATKOWSKI, D.; PHILLIPS, C.B, P.; SCHMIDT, P.; SHIN, Y. Testing the null hypothesis of stationarity against the alternative of a unit root: How sure are we that economic time series have a unit root? Journal of Econometrics, v. 54, n. 0304-4076, p. 159-178, 1992.

LARIAU, A.; EL SAID, M.; TAKEBE, M. An assessment of the exchange rate pass-through in Angola and Nigeria. IMF, 2016. (Working paper, n. 191)

LENDELE, K.; KAMANDA, K-M, J. Nature et spécificité de la dollarisation de l'économie congolaise (RDC). De Boeck Université, v. 130, p. 41-62, 2005.

LOLOH, F. Exchange rate pass-through in Ghana. International Business Research, v. 3, n. 2, p. 186, 2010.

LUTKEPOHL, H. New Introduction to Multiple Time Series Analysis. Springer, 2006.

MABULANGO, J, D.; BOBOY, Y, T. Monetary policy transmission mechanism in DRC: a Bayesian VAR analysis. Lareq, 2016. (Working Paper Series, v. III001)

MALlIC, S.; SOUSA, R. Commodity Prices, Inflationary Pressures, and Monetary Policy: Evidence from BRICS Economies. Open Economies Review, v. 24, n. 4, p. 677-694, 2013.

MELO, A. C. de. Brazil's Commodity Price Index, IFC Bulletin. n. 36, BIS, 2013.

MENGA, L, E. Pass-Through of Exchange rate to inflation in DRC 2002 to 2007. MPRA Paper, n. 21970, 2010.

MOREIRA, R. R. Commodities prices index as a variable determining the consumer inflation rate and the monetary policy: Recent evidences for the Brazilian economy through a VAR analysis. Economics and Finance Review. v. 2, p. 01-09, 2012a.

MOREIRA, R. R. Some notes on a commodities net-exporting economy: exchange rate, consumer inflation and monetary policy. Economics and Finance Review, v. 3, p. 26-30, 2014. 
OREIRO, J. L., PAUlA, L. F., ONO, F. H.; SILVA, G. J. C. Determinantes macroeconômicos do spread bancário no Brasil: teoria e evidência recente. Economia Aplicada, v. 10, n. 4, p. 609-634, 2006.

PEREIRA, R, T.; CARVALHO, A. Desvalorização cambial e seu impacto sobre os custos e preços industriais no brasil: uma análise dos efeitos de encadeamento nos setores produtivos. IPEA, 2000. (Texto para discussão, n. 711)

PESARAN, H.; SHIN, Y. Generalized impulse response analysis in linear multivariate models. Economics Letter, v. 58, n. 1, p. 17-29, 1998.

PHILlIPS, P. C. B; PERRON, P. Testing for a unit root in time series regression. Biometrika, v. 75, n. 2, p. 335-346, 1988.

PINSHI. P, C.; SUNGANI, E. The relevance of pass-through effect: should we revisit monetary policy regime? International Journal of Economics. Business and Management Research, v. 2, n. 2, p. 2456-7760, 2018.

ROSENGREN, E. S. et al. A look inside a key economic debate: how should monetary policy respond to price increases driven by supply shocks? Remarks to the Massachusetts Chapter of NAIOP, the Commercial Real Estate Development Association, v. 4, 2011.

SCHMIDT-HEBBEL, K.; MATÍAS, T. Monetary policy implementation and results in twenty inflation-targeting countries. Banco Central de Chile, v. 166, 2002.

SOLOMON, M.; de WET, A. The Effect of a Budget Deficit on Inflation: The Case of Tanzania. SAJEMS NS, v.7, n. 1, 2004.

SVENSON, E. O. L. Oil Prices and ECB Monetary Policy. Princeton University, CEPR, and NBER, EP501, 2005.

TAYLOR, J. B. Aggregate Dynamics and Staggered Contracts. The Journal of Political Economy, v. 88, n. 1, p. 1-23, 1980.

TAYLOR, J. B. Banco Central do Congo. Relatório de Inflação. Disponível em: $<\mathrm{http}: / / \mathrm{www}$. bcc.cd/index.php?option=com_content $\&$ view=article\&id=159\&Itemid=8 $7>$.

TAYLOR, J. B. Fundo Monetário Internacional. IMF. République démocratique du Congo: Programme de référence: Lettre d'intention, Mémorandum de politique économique et financière, et protocole d'accord technique. Disponível em: <https://www.imf.org/external/np/loi/2009/cod/fra/113009f.pdf>

TAYLOR, J. B. Low inflation, pass-through, and the pricing power of firms. European Economic Review, v. 44, n. 7, p. 1389-1408, 2000. 
TAYLOR, J. B. The Observatory of Economic Complexity: OEC. Disponível em: $<$ https://atlas.media.mit.edu/en/>. Acesso em: 08 ago. 2018. 